\title{
Greater Dietary Inflammatory Index score is associated with higher likelihood of chronic kidney disease
}

\author{
Mohsen Mazidi ${ }^{1,2 *}$, Nitin Shivappa ${ }^{3,4,5}$, Michael D. Wirth ${ }^{3,4,5}$, James R. Hebert ${ }^{3,4,5}$ and Andre P. Kengne ${ }^{6}$ \\ ${ }^{1}$ Key State Laboratory of Molecular Developmental Biology, Institute of Genetics and Developmental Biology, Chinese Academy \\ of Sciences, Beijing 100101, People's Republic of China \\ ${ }^{2}$ Institute of Genetics and Developmental Biology, International College, The University of Chinese Academy of Science, Beijing \\ 100101, People's Republic of China \\ ${ }^{3}$ Cancer Prevention and Control Program, University of South Carolina, 915 Greene Street, Suite 200, Columbia, \\ SC 29208, USA \\ ${ }^{4}$ Department of Epidemiology and Biostatistics, University of South Carolina, 915 Greene Street, Suite 400, Columbia, \\ SC 29208, USA \\ ${ }^{5}$ Connecting Health Innovations, LLC, 1417 Gregg Street, Columbia, SC 29201, USA \\ ${ }^{6}$ Non-Communicable Disease Research Unit, South African Medical Research Council and University of Cape Town, \\ PO Box 19070, Tygerberg, 7505, South Africa
}

(Submitted 26 May 2017 - Final revision received 22 February 2018 - Accepted 7 March 2018)

\section{Abstract}

Chronic kidney disease (CKD) is described as a progressive alteration of kidney function, resulting from multiple factors, including behaviours. We investigated the association of the Dietary Inflammatory Index (DII ${ }^{\circledR}$ ) with prevalent CKD in adult Americans. National Health and Nutrition Examination Survey participants with measured data on kidney function markers from 2005 to 2012 were included in this study. Prevalent CKD was based on an estimated glomerular filtration rate (eGFR) $<60 \mathrm{ml} / \mathrm{min}$ per $1.73 \mathrm{~m}^{2}$ or urinary albumin/creatinine $\geq 30 \mathrm{mg} / \mathrm{g}$. Energy-adjusted DII $\left(\mathrm{E}-\mathrm{DII}{ }^{\mathrm{TM}}\right)$ scores were calculated from 24-h dietary recalls. Statistical analyses accounted for the survey design and sample weights. We included 21649 participants, with 1634 (6.8\%) having prevalent CKD. Participants with high E-DII scores had greater BMI, fasting blood glucose and systolic blood pressure, and were more likely to be diabetic or hypertensive (all $P<0.001$ ) compared with those with lower E-DII scores. In regression models adjusted for age, sex, race, fasting blood glucose, blood pressure, BMI, hypertension and diabetes status, mean eGFR significantly decreased across increasing quartiles of E-DII, whereas serum uric acid level and log urinary albumin:creatinine ratio significantly increased (all $P<0 \cdot 001$ ). Prevalent CKD increased from $5.3 \%$ in the lowest to $9.3 \%$ in the highest E-DII quartile $(P=0.02)$. In multivariable-adjusted logistic regression models, the odds of prevalent CKD were $29 \%$ higher in the highest compared with the lowest E-DII quartile. Pro-inflammatory diet is associated with declining kidney function and high prevalence of CKD. Dietary changes that reduce inflammation have a potential to prevent CKD.

Key words: Dietary Inflammatory Index: Inflammation: Chronic kidney disease: National Health and Nutrition Examination Survey

Chronic kidney disease (CKD) is a progressive loss of kidney function over time, leading to irreversible kidney failure ${ }^{(1)}$. The pathophysiological process involved in CKD is characterised by a background of low-grade chronic inflammation ${ }^{(2)}$. Together with coagulation disorders and neutrophil-endothelium interaction, inflammation is believed to play a role in the genesis of kidney injury, potentially leading to chronically impaired kidney function $^{(3)}$.

Diet may play a central role in the regulation of chronic inflammation $^{(4)}$ and, possibly, in kidney health. Anti-inflammatory nutrients such as $n-3$ PUFA, fibre and many vitamins ${ }^{(5)}$ have been associated with better kidney function, lower risk of albuminuria and slower decline in kidney function ${ }^{(5-7)}$. Conversely, nutrients assumed to have pro-inflammatory effects such as SFA or sugar ${ }^{(8)}$ have been linked with worsening of kidney function ${ }^{(9)}$. Studies have concluded that Dietary Approaches to Stop Hypertension (DASH) and Mediterranean dietary patterns, which are rich in protective nutrients such as antioxidant vitamins including vitamin E, C, A, K, Mg, Ca, fibre, PUFA, MUFA and phytochemicals and poor in SFA, trans-fatty acids and simple carbohydrate, can affect kidney function and decrease the risk of $\mathrm{CKD}^{(10,11)}$.

The Dietary Inflammatory Index (DII ${ }^{\circledR}$ ) was designed to assess the inflammatory potential of the diet based on the proand anti-inflammatory properties of its various components.

Abbreviations: ACR, urinary albumin:creatinine ratio; CKD, chronic kidney disease; DII, Dietary Inflammatory Index; E-DII, energy-adjusted dietary inflammatory index; eGFR, estimate glomerular filtration rate; NHANES, Nutrition and Health Examination Surveys.

* Corresponding author: M. Mazidi, email moshen@genetics.ac.cn 
The DII was developed to provide a means for estimating the overall inflammatory potential of the diet ${ }^{(12,13)}$. Previously, the DII was associated with a range of outcomes including markers of systemic inflammation, CVD, telomere length and overall mortality $^{(14-18)}$. However, there is very limited evidence on the association between dietary patterns and kidney health ${ }^{(19)}$. The main objectives in the management of CKD are the reduction of unfavourable symptoms of uraemia, delaying the start of renal replacement therapy and quality-of-life improvement ${ }^{(20)}$. Diet, specifically through a focus on foods with anti-inflammatory properties, including those with high concentrations of vitamins and minerals, has the potential to decrease oxidative stress. Dietary restrictions imposed in CKD subjects make it difficult to ensure adequate micronutrient content in the diet, while, at the same time, it has been suggested that these patients have an impaired intestinal absorption of minerals and vitamins ${ }^{(21)}$.

The aim of this study was to investigate the association of the inflammatory potential of diet with kidney function and prevalent CKD in adult Americans. We hypothesised that those living with prevalent CKD would have more pro-inflammatory diets than those without CKD.

\section{Methods}

\section{Population}

We used data from the Nutrition and Health Examination Surveys (NHANES), which is described in greater detail elsewhere $^{(22)}$. In brief, these are periodic cross-sectional surveys conducted by the US National Center for Health Statistics, and during which home visits are conducted to administer questionnaires to collect data on demographics, diet and other health behaviours. NHANES applies complex multistage probability sampling procedure to ensure selection of participants from various geographical locations and adequate racial/ethnic representation $^{(22)}$. Trained interviewers collected participants' demographic, socio-economic, dietary and health-related information using questionnaires administered during home visits. Clinical examination and dietary assessment are conducted by skilled personnel using a mobile examination centre $(\mathrm{MEC})^{(22)}$. All procedures were carried out in accordance with relevant approved guidelines and regulations ${ }^{(23-26)}$. Informed consent was obtained from all participants, and the National Centre for Health Statistics Research Ethics Review Board approved the protocol. For the present analysis, four survey cycles (i.e. 2005-2006, 2007-2008, 2009-2010 and 2011-2012) were combined to produce estimates with greater precision and smaller sampling error ( $n$ 40790). The analytical sample was limited to adults aged $\geq 18$ years. After excluding pregnant and lactating women ( $n$ 986), as well as participants with missing information on the variables of interest ( $n$ 1547), the final analytical sample included 21649 respondents from NHANES 2005-2012.

\section{Biochemical analysis}

Methods for Biochemical analyses are described in the NHANES Laboratory/Medical Technologists Procedures Manual ${ }^{(23-26)}$.
A blood specimen was drawn from the participant's antecubital vein by a trained phlebotomist according to a standardised protocol. Fasting glucose was measured in plasma by a hexokinase method using a Roche/Hitachi 911 Analyzer and Roche Modular P Chemistry Analyzer. The concentration of creatinine in serum was determined using the modular chemistry side of a Beckman Coulter DxC800 using the Jaffe reaction method (kinetic alkaline picrate). The creatinine calibration is traceable to an isotope dilution MS reference method ${ }^{(27)}$. Urinary creatinine by the Jaffe rate reaction, and urinary albumin by solid-phase fluorescent immunoassay, from a random urine sample ${ }^{(28)}$ were used to calculate the urinary albumin:creatinine ratio (ACR). The CKD Epidemiology Collaboration equation ${ }^{(29)}$ was used to estimate glomerular filtration rate (eGFR, in $\mathrm{ml} / \mathrm{min}$ per $1.73 \mathrm{~m}^{2}$ ), and eGFR lower than $60 \mathrm{ml} / \mathrm{min}$ per $1.73 \mathrm{~m}^{2}$ was used to define low eGFR. ACR $>30 \mathrm{mg} / \mathrm{g}$ was used to define albuminuria, and the presence of either low eGFR or albuminuria was used to define CKD in line with Kidney Disease: Improving Global Outcomes 2012 recommendations ${ }^{(30)}$.

\section{Diet and Dietary Inflammatory Index}

Dietary data in NHANES were collected using a single 24-h dietary recall interview at the $\mathrm{MEC}^{(22)}$. The development and validation of the DII has been discussed in detail elsewhere ${ }^{(12)}$. The 24-hour-derived dietary information was used to calculate energy-adjusted DII (E-DII ${ }^{\mathrm{TM}}$ ) scores for all participants ${ }^{(12)}$. The DII food parameters available in NHANES database included carbohydrates; protein; fat; grams of alcohol; fibre; cholesterol; SFA, MUFA and PUFA; $n-3$ and $n$-6 PUFA; niacin; vitamins A, $\mathrm{B}_{1}, \mathrm{~B}_{2}, \mathrm{~B}_{6}, \mathrm{~B}_{12}$, C, D and E; Fe; Mg; Zn; Se; folic acid; $\beta$-carotene; and caffeine. Higher (i.e. more positive) scores tend to indicate more pro-inflammatory diets and more negative values are more anti-inflammatory ${ }^{(12)}$. To control for the effect of total energy intake, the E-DII was calculated per $4184 \mathrm{~kJ}$ (1000 kcal) of food consumed. We used energy-adjusted food parameters wherein we calculated all the food parameters per $4184 \mathrm{~kJ}$ (1000 kcal) of consumption. This required using an energyadjusted world database.

\section{Statistical analysis}

Data analyses followed the CDC guidelines for complex NHANES data analysis, accounting for the masked variance and using the recommended weighting methodology ${ }^{(31)}$, implemented with the use of SPSS ${ }^{\circledR}$ complex sample module version 22.0 (IBM Corp). We used means with their standard errors for continuous variables (with groups compared via ANOVA) and percentages for categorical variables (with groups compared using the $\chi^{2}$ test). The Kolmogorov-Smirnov test was used to evaluate the normal distribution of continuous variables. The natural logarithm of ACR and urinary albumin were taken to approximate a normal distribution. Adjusted mean of kidney function markers across E-DII quartiles was calculated using ANCOVA. These models were adjusted for age, sex, race, fasting blood glucose, systolic and diastolic blood pressure, BMI $\left(\mathrm{kg} / \mathrm{m}^{2}\right.$ ), diabetes (self-reported history of diabetes or fasting plasma glucose $\geq 7.0 \mathrm{mmol} / \mathrm{l}(126 \mathrm{mg} / \mathrm{dl})$ ) and hypertension. 
Logistic regressions models, using a similar adjustment strategy, were then used to derive the OR and $95 \%$ CI for the association of E-DII (by quartile) with prevalent CKD, always using the lowest quartile as reference. A $P$ value $<0.05$ was used as the nominal cut-off point to indicate statistically significant results.

\section{Results}

Of the 21649 participants included in the analyses, 1634 (6.8\%) had prevalent CKD. The characteristics of participants overall and across E-DII quartiles are summarised in Table 1. The E-DII score ranged from -5.66 to +4.33 , with a median of 0.44 (25th75 th percentiles (i.e. interquartile range -1.04 to 1.62 ). Mean age decreased from 53.6 to 42.1 years $(P<0.001)$, whereas the proportion of women decreased from $58.3 \%$ to $47 \cdot 1 \%$ $(P<0.001)$ across increasing quartiles of E-DII. Across increasing E-DII quartiles, the proportion of non-Hispanic White (the largest ethnic group) followed a U-shape; the proportions of Mexican-American and other Hispanics followed a reversed U-shape; the proportions of non-Hispanic Blacks increased; and the proportion of the remaining racial groups decreased $(P<0.001)$. The proportion of participants with more than high school education (the larger group) followed a reversed U-shape, those with high school education (the second larger group) followed a U-shape pattern and participants with less than high school education decreased from $16 \cdot 8 \%$ in the lowest quartile to $8 \%$ in the top quartile of the E-DII distribution $(P<0.001)$. The cardio-metabolic risk profile systematically deteriorated across increasing quartiles of the E-DII (all $P<0 \cdot 001$ ). For instance, values (highest $v$. lowest E-DII quartiles) were $5.66 v .5 .48 \mathrm{mmol} / \mathrm{l}$ (102 $v$. $98.7 \mathrm{mg} / \mathrm{dl}$ ) for mean fasting blood glucose, $124.2 v .121 .6 \mathrm{mmHg}$ for systolic blood pressure, $29 \cdot 2 v \cdot 28 \cdot 2 \mathrm{~kg} / \mathrm{m}^{2}$ for BMI, $11.2 v .8 \cdot 0 \%$ for prevalent diabetes mellitus and $34 \cdot 1 v$. $28.1 \%$ for prevalent hypertension, as shown in Table 1.

Adjusted mean levels of kidney function markers by quartile of E-DII score are shown in Table 2. Across increasing E-DII quartiles, mean serum uric acid increased from 316 to 329 $\mu \mathrm{mol} / \mathrm{l}(5.31$ to $5.53 \mathrm{mg} / \mathrm{dl})(P<0.001)$, urine albumin increased from $2 \cdot 01$ to $2 \cdot 25 \mathrm{mg} / \mathrm{l})(P<0 \cdot 001)$ and eGFR decreased from 96.3 to $90.7 \mathrm{ml} / \mathrm{min} / 1.73 \mathrm{~m}^{2} \quad(P<0.001)$. Log ACR also increased from $2 \cdot 10$ to $2 \cdot 19(P=0.023)$. The proportion of participants with prevalent low eGFR, albuminuria or CKD systematically increased across increasing quartiles of E-DII (all $P<0.001$ for linear trend). Proportions of participants with CKD, by E-DII quartile, were $11.2 \%$ in the first (lowest) quartile, $12.1 \%$ in the second quartile, $14.1 \%$ in the third quartile and $16.4 \%$ in the top quartile (Table 1 ). In age-, sex- and raceadjusted logistic regressions, compared with the lowest quartile of the E-DII, the OR was 1.14 (95\% CI $0.95,1.37$ ) for the second quartile, 1.24 (95\% CI 1.02, 1.50) for the third quartile and 1.35 (95\% CI $1.08,1.69)$ for the top quartile $\left(P_{\text {for trend }}<0.001\right.$, CKD diagnosed by eGFR). In logistic regression models adjusted for age, sex, race, blood glucose, blood pressure, BMI, diabetes and

Table 1. Descriptive characteristics of participants across quartiles of energy-adjusted Dietary Inflammatory Index (E-DII) (Mean values with their standard errors)

\begin{tabular}{|c|c|c|c|c|c|c|c|c|c|}
\hline \multirow[b]{3}{*}{ Characteristics } & \multicolumn{8}{|c|}{ Quartiles of E-DII } & \multirow[b]{3}{*}{$P$} \\
\hline & \multicolumn{2}{|c|}{ First } & \multicolumn{2}{|c|}{ Second } & \multicolumn{2}{|c|}{ Third } & \multicolumn{2}{|c|}{ Fourth } & \\
\hline & Mean & SEM & Mean & SEM & Mean & SEM & Mean & SEM & \\
\hline Min. and max. of E-DII & \multicolumn{2}{|c|}{-5.39 to -1.06} & \multicolumn{2}{|c|}{-1.07 to 0.39} & \multicolumn{2}{|c|}{0.38 to 1.74} & \multicolumn{2}{|c|}{1.75 to 4.62} & \\
\hline Number of participants & \multicolumn{2}{|c|}{5153} & \multicolumn{2}{|c|}{5131} & \multicolumn{2}{|c|}{5147} & \multicolumn{2}{|c|}{5128} & \\
\hline $\operatorname{Sex}(\%)$ & & & & & & & & & $<0.001$ \\
\hline Male & \multicolumn{2}{|c|}{$41 \cdot 7$} & \multicolumn{2}{|c|}{$48 \cdot 6$} & \multicolumn{2}{|c|}{$52 \cdot 7$} & \multicolumn{2}{|c|}{52.9} & \\
\hline Female & \multicolumn{2}{|c|}{$58 \cdot 3$} & \multicolumn{2}{|c|}{51.4} & \multicolumn{2}{|c|}{$47 \cdot 3$} & \multicolumn{2}{|c|}{$47 \cdot 1$} & \\
\hline Age (years) & $53 \cdot 6$ & 0.23 & $48 \cdot 8$ & 0.25 & $44 \cdot 7$ & 0.26 & $42 \cdot 1$ & 0.25 & $<0.001$ \\
\hline Race (\%) & & & & & & & & & $<0.001$ \\
\hline White (non-Hispanic) & \multicolumn{2}{|c|}{$46 \cdot 7$} & \multicolumn{2}{|c|}{43.4} & \multicolumn{2}{|c|}{$43 \cdot 1$} & \multicolumn{2}{|c|}{$46 \cdot 4$} & \\
\hline Non-Hispanic Black & \multicolumn{2}{|c|}{$16 \cdot 8$} & \multicolumn{2}{|c|}{$19 \cdot 2$} & \multicolumn{2}{|c|}{23.8} & & & \\
\hline Mexican-American & & & & & & & & & \\
\hline Other Hispanic & & & & & & & & & \\
\hline Other & & & & & & & & & \\
\hline Education (\%) & & & & & & & & & $<0.001$ \\
\hline Less than high school & & & & & & & & & \\
\hline Completed high school & & & & & & & & & \\
\hline More than high school & & & & & & & & & \\
\hline Fasting blood glucose $(\mathrm{mg} / \mathrm{dl})^{\star}$ & $98 \cdot 7$ & 0.42 & $100 \cdot 1$ & 0.39 & $102 \cdot 8$ & 0.49 & $102 \cdot 4$ & 0.52 & $<0.001$ \\
\hline Systolic blood pressure $(\mathrm{mmHg})$ & $121 \cdot 6$ & 0.29 & $122 \cdot 6$ & 0.43 & $123 \cdot 3$ & 0.62 & $124 \cdot 2$ & 0.27 & $<0.001$ \\
\hline Diastolic blood pressure $(\mathrm{mmHg})$ & $68 \cdot 4$ & 0.17 & $69 \cdot 1$ & 0.11 & 69.5 & 0.19 & $69 \cdot 7$ & 0.32 & $<0.001$ \\
\hline BMI $\left(\mathrm{kg} / \mathrm{m}^{2}\right)$ & $28 \cdot 2$ & 0.08 & $28 \cdot 7$ & 0.04 & 28.9 & 0.09 & $29 \cdot 2$ & $0 \cdot 10$ & $<0.001$ \\
\hline Type 2 diabetes (\%) & & & & & & & & & $<0.001$ \\
\hline Hypertension (\%) & & & & & & & & & $<0.001$ \\
\hline Low eGFR (\%) & & & & & & & & & $<0.001$ \\
\hline Albuminuria (\%) & & & & & & & & & $<0.001$ \\
\hline CKD (\%) (low eGFR or albuminuria) & & & & & & & & & $<0.001$ \\
\hline
\end{tabular}

eGFR, estimate glomerular filtration rate; CKD, chronic kidney disease.

* To convert glucose in $\mathrm{mg} / \mathrm{dl}$ to $\mathrm{mmol} / \mathrm{l}$, multiply by 0.0555 . 
Table 2. Adjusted (age, sex, race, fasting blood glucose, systolic and diastolic blood pressure, BMI, diabetes and hypertension) mean levels of markers of chronic kidney disease (CKD) across quartiles of energy-adjusted Dietary Inflammatory Index (E-DII)

(Mean values with their standard errors)

\begin{tabular}{|c|c|c|c|c|c|c|c|c|c|}
\hline \multirow[b]{3}{*}{ Characteristics } & \multicolumn{8}{|c|}{ Quartiles of E-DII } & \multirow[b]{3}{*}{$P$} \\
\hline & \multicolumn{2}{|c|}{ First } & \multicolumn{2}{|c|}{ Second } & \multicolumn{2}{|c|}{ Third } & \multicolumn{2}{|c|}{ Fourth } & \\
\hline & Mean & SEM & Mean & SEM & Mean & SEM & Mean & SEM & \\
\hline Number of participants & \multicolumn{2}{|c|}{5153} & \multicolumn{2}{|c|}{5131} & \multicolumn{2}{|c|}{5147} & \multicolumn{2}{|c|}{5128} & \\
\hline Log urine albumin (mg/l) & $2 \cdot 01$ & 0.02 & $2 \cdot 11$ & 0.02 & $2 \cdot 17$ & 0.02 & $2 \cdot 25$ & 0.02 & $<0.001$ \\
\hline Serum creatinine $(\mathrm{mg} / \mathrm{dl})^{*}$ & 0.88 & 0.006 & 0.89 & 0.005 & 0.89 & 0.004 & 0.90 & 0.003 & 0.19 \\
\hline Serum uric acid $(\mathrm{mg} / \mathrm{dl})^{\star}$ & $5 \cdot 31$ & 0.26 & $5 \cdot 42$ & 0.37 & $5 \cdot 48$ & 0.41 & 5.53 & 0.62 & $<0.001$ \\
\hline $\log$ ACR (mg/g) & $2 \cdot 10$ & 0.01 & $2 \cdot 11$ & 0.02 & $2 \cdot 11$ & 0.02 & $2 \cdot 19$ & 0.02 & 0.023 \\
\hline eGFR $\left(\mathrm{ml} / \mathrm{min}\right.$ per $\left.1.73 \mathrm{~m}^{2}\right)$ & $96 \cdot 26$ & 0.52 & 95.48 & 0.48 & $92 \cdot 47$ & 0.61 & 90.71 & 0.82 & $<0.001$ \\
\hline
\end{tabular}

eGFR, estimated glomerular filtration rate, ACR, urine albumin:creatinine ratio.

* To convert creatinine in $\mathrm{mg} / \mathrm{dl}$ to $\mu \mathrm{mol} / \mathrm{l}$, multiply by 88.4 ; to convert uric acid in $\mathrm{mg} / \mathrm{dl}$ to $\mu \mathrm{mol} / \mathrm{l}$, multiply by 59.48 .

hypertension status, compared with the lowest quartile of E-DII, the OR of low eGFR was 1.09 (95\% CI $0.90,1.32$ ) for the second quartile, 1.18 (95\% CI $0.97,1.44)$ for the third quartile and 1.29 (95\% CI $1.03,1.62)$ for the top quartile $\left(P_{\text {for trend }}<0.001\right)$. Equivalent figures were $1.11(95 \% \mathrm{CI} 0.78,1.59), 1.13(95 \% \mathrm{CI}$ $1.06,1.22)$ and $1.19(95 \% \mathrm{CI} 1.10,1.28)$ for albuminuria $\left(P_{\mathrm{for}}\right.$ trend $<0.001)$, and $1.08(95 \%$ CI $1.02,1 \cdot 13), 1 \cdot 15$ (95\% CI 1.08 , $1.23)$ and $1.23(95 \%$ CI $1.10,1.35)$ for CKD $\left(P_{\text {for trend }}<0.001\right)$

\section{Discussion}

This study examined the association between the E-DII and prevalent CKD in a large population of adult Americans. A proinflammatory diet was associated with adverse profiles of kidney function markers, translating into higher rates of prevalent CKD, even after adjustment for a range of extraneous factors.

In line with our findings, recent investigations have reported that a pro-inflammatory diet was associated with systemic inflammation, as well as with reduced kidney function, in elderly individuals ${ }^{(32)}$. In the prospective observational Nurses' Health Study, it was reported that, compared with the lowest quartile, the highest quartile of Western pattern score 'proinflammatory' was associated with a high risk of microalbuminuria (OR 2.17; 95\% CI 1.18, 3.66) and rapid eGFR decline $\geq 3 \mathrm{ml} / \mathrm{min}$ per $1.73 \mathrm{~m}^{2}$ per year (OR $1.77 ; 95 \%$ CI 1.03 , $3.03)^{(33)}$. On the other hand, women in the top quartile of the DASH score (highly loaded with anti-inflammatory materials such as fruit and vegetables) had decreased risk of rapid eGFR decline (OR 0.55; 95\% CI 0.38, 0.80) (33) $^{(3)}$

An investigation in the Multi-ethnic Study of Atherosclerosis that included almost 5000 ethnically diverse men and women reported that a dietary pattern rich in whole grains and fruit (anti-inflammatory) was associated with lower urinary ACR (20\% lower ACR across quintiles, $P=0.004$ ), whereas animalbased food (pro-inflammatory) intake was directly associated $11 \%$ higher ACR across quintiles $(P=0 \cdot 03)^{(34)}$. We previously reported that higher dietary intake of animal fat was associated with the presence of microalbuminuria, whereas higher sodium intake was directly associated and higher $\beta$-carotene intake was inversely associated with faster eGFR decline over 11 years ${ }^{(35)}$. In the same line, the 'anti-inflammatory' Healthy Eating Index ${ }^{(36)}$ was associated with a lower risk of albuminuria and
eGFR decline in diabetic people ${ }^{(5,32)}$. In another observational study conducted among 1942 elderly community-dwelling participants aged 70-71 years, diet with higher pro-inflammatory load was associated with reduced kidney function ${ }^{(32)}$.

It has been suggested that poor dietary quality could lead to several tissue-specific and systemic metabolic dysfunctions that could promote renal dysfunction ${ }^{(37,38)}$. For example, it has been reported that diets high in whole grains, fruit or vegetables and fish (anti-inflammatory) are inversely associated with markers of inflammation including CRP and soluble ICAM-1, whereas a diet pattern rich in fats and processed meats (pro-inflammatory) is directly associated with markers of inflammation including $\mathrm{CRP}^{(36)}$. Furthermore, cytokine-mediated inflammation has been suggested to be involved in the early stages of impaired kidney function in the elderly, whereas cyclooxygenasemediated inflammation does not appear to play a role at this stage $^{(39)}$. Thus, inflammation seems to be a reasonable target for potential preventive and therapeutic interventions in patients with CKD.

\section{Strengths and limitations}

This is among the largest studies on the association of kidney disease with E-DII. Participants were a random sample of the general population, and therefore the results can be extrapolated to the general US population. Because data collection was performed on all days of the week in NHANES, the potential for dayspecific information bias is very low ${ }^{(40,41)}$. Our findings also have to be considered in the context of some study limitations. First, the cross-sectional nature of the data does not allow for direct inference about causality. Second, it is well known that a single 24-h diet recall interview is not ideal for characterising an individual's long-term habitual intake ${ }^{(42,43)}$. Third, calculation of E-DII scores was based on twenty-seven out of forty-five food parameters, which may affect our findings. Despite these shortcomings, which would have increased overall error, we were able to detect a relationship between E-DII scores and CKD.

\section{Conclusion}

The potentially deleterious effect of pro-inflammatory diet on kidney health, supported by our findings and those of other 
investigators, suggests the potential utility of the modulation of inflammatory properties of diet, in strategies to prevent kidney disease. If confirmed in clinical trial, this knowledge may have application for both population-wide and high-risk approach to CKD prevention and control in various settings.

\section{Acknowledgements}

M. M. was supported by The world academy of science and Chinese Academy of Sciences. Drs N. S., M. D. W. and J. R. H. were supported by grant no. R44DK103377 to Connecting Health Innovations LLC (CHI) from the US National Institute of Diabetes and Digestive and Kidney Diseases.

M. M. was involved in study conception, data analysis and interpretation and drafting of the manuscript; N. S. contributed to data analysis and interpretation and critical revision of the manuscript; M. D. W. performed data analysis and interpretation and critical revision of the manuscript; J. R. H. performed data analysis and interpretation and critical revision of the manuscript; A. P. K. contributed to data interpretation and critical revision of the manuscript.

Dr J. R. H. owns controlling interest in CHI, a company planning to license the right to his invention of the Dietary Inflammatory Index $\left(\mathrm{DII}^{\circledR}\right)$ from the University of South Carolina in order to develop computer and smart phone applications for patient counselling and dietary intervention in clinical settings. Drs N. S. and M. D. W. are employees of CHI.

The authors declare that there are no conflicts of interest.

\section{References}

1. Levey AS \& Coresh J (2012) Chronic kidney disease. Lancet 379, 165-180.

2. Miyamoto T, Carrero JJ \& Stenvinkel P (2011) Inflammation as a risk factor and target for therapy in chronic kidney disease. Curr Opin Nephrol Hypertens 20, 662-668.

3. Leung KC, Tonelli M \& James MT (2013) Chronic kidney disease following acute kidney injury-risk and outcomes. Nat Rev Nephrol 9, 77-85.

4. Giugliano D, Ceriello A \& Esposito K (2006) The effects of diet on inflammation: emphasis on the metabolic syndrome. $J \mathrm{Am}$ Coll Cardiol 48, 677-685.

5. Dunkler D, Dehghan M, Teo KK, et al. (2013) Diet and kidney disease in high-risk individuals with type 2 diabetes mellitus. JAMA Intern Med 173, 1682-1692.

6. Miller ER 3rd, Juraschek SP, Appel LJ, et al. (2009) The effect of $n$-3 long-chain polyunsaturated fatty acid supplementation on urine protein excretion and kidney function: meta-analysis of clinical trials. Am J Clin Nutr 89, 1937-1945.

7. Xu H, Huang X, Riserus U, et al. (2014) Dietary fiber, kidney function, inflammation, and mortality risk. Clin J Am SoC Nephrol 9, 2104-2110.

8. Huang X, Sjogren P, Arnlov J, et al. (2014) Serum fatty acid patterns, insulin sensitivity and the metabolic syndrome in individuals with chronic kidney disease. J Intern Med 275 , $71-83$.

9. Lin J, Judd S, Le A, et al. (2010) Associations of dietary fat with albuminuria and kidney dysfunction. Am J Clin Nutr $\mathbf{9 2}$, 897-904.

10. Farhadnejad H, Asghari G, Mirmiran P, et al. (2016) Micronutrient intakes and incidence of chronic kidney disease in adults: Tehran Lipid and Glucose Study. Nutrients 8, 217.
11. Huang X, Jiménez-Moleón JJ, Lindholm B, et al. (2013) Mediterranean diet, kidney function, and mortality in men with CKD. Clin J Am Soc Nephrol 8, 1548-1555.

12. Shivappa N, Steck SE, Hurley TG, et al. (2014) Designing and developing a literature-derived, population-based dietary inflammatory index. Public Health Nutr 17, 1689-1696.

13. Cavicchia PP, Steck SE, Hurley TG, et al. (2009) A new dietary inflammatory index predicts interval changes in serum highsensitivity C-reactive protein. J Nutr 139, 2365-2372.

14. Wirth MD, Burch J, Shivappa N, et al. (2014) Association of a dietary inflammatory index with inflammatory indices and metabolic syndrome among police officers. J Occup Environ Med 56, 986-989.

15. Garcia-Arellano A, Ramallal R, Ruiz-Canela M, et al. (2015) Dietary Inflammatory Index and Incidence of Cardiovascular Disease in the PREDIMED Study. Nutrients 7, 4124-4138.

16. Garcia-Calzon S, Zalba G, Ruiz-Canela M, et al. (2015) Dietary inflammatory index and telomere length in subjects with a high cardiovascular disease risk from the PREDIMEDNAVARRA study: cross-sectional and longitudinal analyses over 5 y. Am J Clin Nutr 102, 897-904.

17. Shivappa N, Steck SE, Hurley TG, et al. (2014) A populationbased dietary inflammatory index predicts levels of C-reactive protein in the Seasonal Variation of Blood Cholesterol Study (SEASONS). Public Health Nutr 17, 1825-1833.

18. Neufcourt L, Assmann KE, Fezeu LK, et al. (2015) Prospective association between the dietary inflammatory index and metabolic syndrome: findings from the SU.VI.MAX study. Nutr Metab Cardiovasc Dis 25, 988-996.

19. Sabatino A, Regolisti G, Gandolfini I, et al. (2017) Diet and enteral nutrition in patients with chronic kidney disease not on dialysis: a review focusing on fat, fiber and protein intake. J Nephrol 30, 743-754.

20. Deniz Ayli M, Ayli M, Ensari C, et al. (2000) Effect of lowprotein diet supplemented with keto acids on progression of disease in patients with chronic renal failure. Nephron $\mathbf{8 4}$, 288-289.

21. Vaziri ND, Said HM, Hollander D, et al. (1985) Impaired intestinal absorption of riboflavin in experimental uremia. Nephron 41, 26-29.

22. Mazidi M, Kengne AP \& Vatanparast H (2018) Association of dietary patterns of American adults with bone mineral density and fracture. Public Health Nutr (epublication ahead of print version 21 May 2018)

23. Needham BL, Adler N, Gregorich S, et al. (2013) Socioeconomic status, health behavior, and leukocyte telomere length in the National Health and Nutrition Examination Survey, 1999-2002. Soc Sci Med 85, 1-8.

24. Remer T (2001) Influence of nutrition on acid-base balance metabolic aspects. Eur J Nutr 40, 214-220.

25. Mazidi M, Michos ED \& Banach M (2017) The association of telomere length and serum 25-hydroxyvitamin D levels in US adults: the National Health and Nutrition Examination Survey. Arch Med Sci 13, 61-65.

26. Mazidi M, Gao HK \& Kengne AP (2018) Lipid accumulation product and visceral adiposity index are associated with dietary patterns in adult Americans. Medicine (Baltimore) 97, e0322.

27. Selvin E, Manzi J, Stevens LA, et al. (2007) Calibration of serum creatinine in the National Health and Nutrition Examination Surveys (NHANES) 1988-1994, 1999-2004. Am J Kidney Dis 50, 918-926.

28. Chavers BM, Simonson J \& Michael AF (1984) A solid phase fluorescent immunoassay for the measurement of human urinary albumin. Kidney Int 25, 576-578. 
29. Levey AS, Stevens LA, Schmid CH, et al. (2009) A new equation to estimate glomerular filtration rate. Ann Intern Med 150, 604-612.

30. Kidney Disease: Improving Global Outcomes (2013) Chapter 1: definition and classification of CKD. Kidney Int Suppl (2011) 3, 19-62.

31. Mazidi M, Mikhailidis DP \& Banach M (2018) Higher dietary acid load is associated with higher likelihood of peripheral arterial disease among American adults. J Diabetes Complications 32, 565-569.

32. Xu H, Sjögren P, Ärnlöv J, et al. (2015) A proinflammatory diet is associated with systemic inflammation and reduced kidney function in elderly adults. J Nutr 145, 729-735.

33. Lin J, Fung TT, Hu FB, et al. (2011) Association of dietary patterns with albuminuria and kidney function decline in older white women: a subgroup analysis from the Nurses' Health Study. Am J Kidney Dis 57, 245-254.

34. Nettleton JA, Steffen LM, Palmas W, et al. (2008) Associations between microalbuminuria and animal foods, plant foods, and dietary patterns in the Multiethnic Study of Atherosclerosis. Am J Clin Nutr 87, 1825-1836.

35. Lin J, Hu FB \& Curhan GC (2010) Associations of diet with albuminuria and kidney function decline. Clin J Am SoC Nephrol 5, 836-843.

36. Nettleton JA, Steffen LM, Mayer-Davis EJ, et al. (2006) Dietary patterns are associated with biochemical markers of inflammation and endothelial activation in the Multi-Ethnic Study of Atherosclerosis (MESA). Am J Clin Nutr 83, 1369-1379.

37. Gopinath B, Harris D, Flood V, et al. (2013) A better diet quality is associated with a reduced likelihood of CKD in older adults. Nutr Metab Cardiovasc Dis 23, 937-943.

38. Odermatt A (2011) The Western-style diet: a major risk factor for impaired kidney function and chronic kidney disease. $A m J$ Physiolo Renal Physiol 301, F919-F931.

39. Nerpin E, Helmersson-Karlqvist J, Risérus U, et al. (2012) Inflammation, oxidative stress, glomerular filtration rate, and albuminuria in elderly men: a cross-sectional study. BMC Res Notes 5, 537.

40. Tooze JA, Midthune D, Dodd KW, et al. (2006) A new statistical method for estimating the usual intake of episodically consumed foods with application to their distribution. $\mathrm{J} \mathrm{Am}$ Diet Assoc 106, 1575-1587.

41. Guenther PM, Ding EL \& Rimm EB (2013) Alcoholic beverage consumption by adults compared to dietary guidelines: results of the National Health and Nutrition Examination Survey, 2009-2010. J Acad Nutr Diet 113, 546-550.

42. Hebert JR, Hurley TG, Steck SE, et al. (2014) Considering the value of dietary assessment data in informing nutrition-related health policy. Adv Nutr 5, 447-455.

43. Ma Y, Olendzki BC, Pagoto SL, et al. (2009) Number of 24-hour diet recalls needed to estimate energy intake. Ann Epidemiol 19, 553-559. 